Article

\title{
Assessment of the Release Profile of Fibroblast Growth Factor-2-Load Mesoporous Calcium Silicate/Poly- $\varepsilon$-caprolactone 3D Scaffold for Regulate Bone Regeneration
}

\author{
Chia-Tze Kao ${ }^{1,2,+}$, Yen-Jen Chen ${ }^{3,4,+}$, Tsui-Hsien Huang ${ }^{1,2}$, Yen-Hong Lin ${ }^{5}$, Tuan-Ti Hsu ${ }^{6, *}$ and \\ Chia-Che Ho ${ }^{7,8, *(\mathbb{D})}$ \\ 1 School of Dentistry, Chung Shan Medical University, Taichung 40447, Taiwan; ctk@csmu.edu.tw (C.-T.K.); \\ thh@csmu.edu.tw (T.-H.H.) \\ 2 Department of Stomatology, Chung Shan Medical University Hospital, Taichung 40447, Taiwan \\ 3 School of Medicine, China Medical University, Taichung City 40447, Taiwan; yenjenc.tw@yahoo.com.tw \\ 4 Department of Orthopedics, China Medical University Hospital, Taichung 40447, Taiwan \\ 5 The Ph.D. Program for Medical Engineering and Rehabilitation Science, China Medical University, \\ Taichung 40447, Taiwan; roger.lin0204@gmail.com \\ 6 x-Dimension Center for Medical Research and Translation, China Medical University Hospital, \\ Taichung 40447, Taiwan \\ 7 Department of Bioinformatics and Medical Engineering, Asia University, Taichung 40447, Taiwan \\ 8 3D Printing Medical Research Institute, Asia University, Taichung 40447, Taiwan \\ * Correspondence: sethho@asia.edu.tw (C.-C.H.); nakohsu@gmail.com (T.-T.H.); \\ Tel.: +886-4-22052121 (C.-C.H.); Fax: +886-4-24759065 (C.-C.H.) \\ + These authors contributed equally to this work.
}

Received: 3 September 2020; Accepted: 1 October 2020; Published: 3 October 2020

check for updates

\begin{abstract}
Recent advances in three-dimensional printing technology enable facile and on-demand fabrication of patient-specific bone scaffolds. However, there is still an urgent need for printable biomaterials with osteoinductivity. In the present study, we propose an approach to synthesize fibroblast growth factor-2 loaded-mesoporous calcium silicate nanoparticles. The growth factor loaded-nanoparticles served as fillers of polycaprolactone and then the composite scaffolds with a controlled pore structure were obtained through a fused deposition modeling technique. To evaluate the feasibility of the composite scaffolds in bone tissue engineering, drug release kinetic, bioactivity, cell proliferation, differentiation, and animal study were conducted. Our findings illustrate that utilization of mesoporous calcium silicate allowed the introduction of fibroblast growth factor- 2 into the composite scaffolds through a simple soaking process and then gradually released from the scaffold to facilitate proliferation and osteogenesis differentiation of human Wharton's jelly mesenchymal stem cells. Additionally, the in vivo femur defect experiments also indicate that the co-existence of calcium silicate and fibrous growth factor- 2 synergistically accelerated new bone formation. These results demonstrate that the fibroblast growth factor-2-loaded mesoporous calcium silicate nanoparticles/polycaprolactone composite scaffolds may serve as potential bone grafts for facilitating repair of defected bone tissues.
\end{abstract}

Keywords: mesoporous calcium silicate nanoparticle; drug delivery; fibroblast growth factor-2; bone tissue engineering; 3D printing 


\section{Introduction}

Repairing critical bone defects always requires the use of implants, such as autologous bone, the allogeneic bone graft, or artificial, metal and ceramic substitutes in clinical settings [1-3]. For the treatment of critical bone defects, novel strategies to solve these challenges are warranted $[4,5]$. Accumulating research in the realm of tissue engineering has delivered a wide spectrum of manufacturing approaches used to fabricate artificial bone grafts in the form of open-porous scaffolds by using degradable biomaterials [6,7]. Among existing technologies, additive manufacturing, also known as three dimensional (3D) printing technologies, have been considered as prominent candidates for producing patient-specific bone scaffolds due to their flexibility and accuracy in macroscale morphology and microscale internal structure controls and are considered promising for predominate manufacturing of clinically available orthopedic implants [8]. To accommodate the clinical requirements of 3D printed-bone scaffolds, thermoplastic polyesters have shown their applicability in bone tissue engineering due to their degradability, bioresorbability, biocompatibility, as well as printability [9]. Particularly, polycaprolactone (PCL) emerges as the most frequently used semicrystalline aliphatic polymer for 3D printed-bone scaffolds considering its lower melting point (about $60^{\circ} \mathrm{C}$ ) and more superior mechanical strength in comparison to others (e.g., polylactic acid) $[10,11]$. Unfortunately, the hydrophobic nature of PCL can impair effective cell-material interactions and hydrolysis, which results in poor healing efficiency and extremely slow degradation rate, consequently impeding successful implementation in bone tissue engineering $[12,13]$.

Efforts have been made that aim to overcome this issue through performing surface modifications to alter surface properties of PCL scaffolds. Gupta et al. demonstrated that poor cell adhesion of PCL scaffolds could be remarkably improved by alkaline etching in concentrated sodium hydroxide [14]. In the meantime, however, the alkaline etching treatment also significantly diminished mechanical strengths of the scaffolds, which may lead to concerns of insufficient mechanical stability and premature failure. Additionally, poor osteogenicity of PCL is another concern that could jeopardize translation of $3 \mathrm{D}$ printed-scaffold from bench to bed. It is still challenging to fulfill multiple clinical requirements with solitary biomaterial designs. In contrast, it can be more flexible to minimize the unmet demands if composites are considered since they provide high freedom to tailor physicochemical and biological properties of the scaffolds [15]. Regarding this, calcium phosphate-based (CP) bioceramics have been proven as effective fillers for improving mechanical strengths, degradation rates, and osteoconductivity of PCL scaffolds [16]. Recently, calcium silicate-based (CS) bioceramics have emerged as popular alternatives to $\mathrm{CP}$ for applications of hard tissue regeneration because of their excellent bioactivity, biocompatibility, and osteogenicity [17]. Additionally, evidence has suggested that degraded ionic products (calcium and silicate ions) from CS-based materials were considered effective for stimulating cell behaviors, such as proliferation, osteogenic differentiation, and mineralization of bone-related progenitor cells $[18,19]$. More importantly, these advantageous characteristics can be retained in the CS/PCL composite scaffolds to facilitate in vitro and in vivo bone regeneration [20,21]. Despite the noticeable osteoconductivity of CS and its composites, attempts intended for developing novel bioceramic fillers with elevated osteoinductivity to optimize bone healing efficiency and clinical availability of composite scaffolds are continuously ongoing [21].

The basic fibroblast growth factor (bFGF), also known as FGF-2, is a versatile biomolecule that can participate in various physiological processes and offers biological cues to regulate cell growth, apoptosis, differentiation, extracellular matrix remodeling, and angiogenesis [22]. Hurley and coworkers demonstrated that deletion of the FGF-2 gene could lead to diminished bone mass and impaired bone formation in mice, indicating an essential role in bone function. Liang et al. indicated that stimulated bone formation was achieved in ovariectomized rats with periodic administration of exogenous bFGF [23]. However, the in vivo half-life of exogenous bFGF is quite short (3-10 $\mathrm{min})$, which can negatively impact its therapeutic efficacy on defected bone tissues [24]. Administration of a high-dose exogenous bFGF is not encouraged because it could turn the stimulant role of bFGF into a depressant one in bone formation [22]. Fortunately, these issues can be addressed through 
employing drug release carriers to protect bFGF from rapid degradation in vivo, as well as achieving long-term controlled release of bFGF with effective and safe dosages. Additionally, synergistic effects on enhancements in osteogenicity of the 3D-printed scaffolds could be achieved due to the coexistence of osteoconductive matters and osteogenic cues in the composites. In the case of CS/PCL scaffolds, both components could be utilized as carriers of bFGF but CS was more preferable than PCL due to the fact that hazard organic solvents should be involved to incorporate bFGF into PCL matrices [25]. To elevate the drug loading capacity, numerous pieces of research have exploited micelle-templated approaches to fabricate ceramic nanoparticles with well-aligned mesoporous channels, which enabled more available area to adsorb drug molecules [26,27]. Our previous results indicate that mesoporous CS nanoparticles with a diameter of about $200 \mathrm{~nm}$ were successfully synthesized and could serve as carriers of bone morphogenic protein-2 (BMP-2) and subsequently stimulated proliferation and osteogenic differentiation of human mesenchymal stem cells (hMSCs) cultured on the 3D printed-scaffolds composed by BMP-2-loaded mesoporous CS and PCL [28].

Therefore, the primary aim of this study was to evaluate the effects of FGF-2-loaded mesoporous CS nanoparticles on the physicochemical properties and osteogenicity of the 3D-printed composite scaffolds. The loading capacity and release profile of the composite scaffolds was determined. The phase composition of the 3D-printed scaffolds before and after being soaked in a cell culture medium was conducted to assess the in vitro apatite forming ability of the scaffolds. The effects of FGF-2 on the in vitro osteogenicity of human Wharton's jelly mesenchymal stem cells (hWJMSCs) cultured on scaffolds with different loading amounts of FGF-2 were examined. Additionally, an animal study was performed to evaluate the efficiency of the composite scaffolds on bone healing in the rabbit femur defect model.

\section{Materials and Methods}

\subsection{CS Mesoporous Nanoparticles Synthesis}

The CS mesoporous nanoparticles were synthesized according to the procedures established by our previous works $[27,29]$. Briefly, $0.6 \mathrm{~g}$ of cetyltrimethylammonium bromide (CTAB, Sigma-Aldrich, St. Louis, MO, USA) and $1 \mathrm{~mL}$ of $\mathrm{NH}_{3} \cdot \mathrm{H}_{2} \mathrm{O}$ were dissolved in $60 \mathrm{~mL}$ of double-distilled water through vigorously stirring at $60^{\circ} \mathrm{C}$ for $20 \mathrm{~min}$. Subsequently, $3 \mathrm{~mL}$ of tetraethyl orthosilicate (TEOS, Sigma-Aldrich) and $3 \mathrm{~g}$ of calcium nitrate (Sigma-Aldrich) were introduced into the solution and stirred for another $3 \mathrm{~h}$. Afterwards, the solution was filtrated and the precipitate was washed three times with $1 \mathrm{~N}$ hydrochloric acid and ethanol alternatively. After being dried at $60^{\circ} \mathrm{C}$, the precipitate powders were sintered at $800^{\circ} \mathrm{C}$ for $2 \mathrm{~h}$. The morphology and microstructure of the CS mesoporous nanoparticles (MCS) were visualized by using a transmission electron microscopy (TEM, JEOL, Tokyo, Japan). The obtained TEM images were used to calculate the sizes of nanoparticles and mesopores by using ImageJ software.

\subsection{FGF-2 Loading}

$1 \mathrm{~g}$ of MCS powder was added to an aqueous solution containing FGF-2 $(5$ or $10 \mu \mathrm{g} / \mathrm{mL}$, named as MCSF5 or MCSF10, respectively) and shaken at room temperature overnight. The FGF-2-loaded MCS (MCSF) powder was isolated from the solution by centrifugation at 15,000 rpm for $30 \mathrm{~min}$. The FGF-2 concentration in the supernatant was analyzed using enzyme-linked immunosorbent assay kit (Invitrogen, Waltham, MA, USA) according to manufacturer's instructions for calculating the loading capacity of MCS powder and the results were obtained in triplicate from three independent tests. Finally, the MCSF powders were washed three times with $\mathrm{ddH}_{2} \mathrm{O}$. After being freeze-dried, the powders were stored at $-20^{\circ} \mathrm{C}$ until further experiments. 


\subsection{MCS and MCSF Scaffold Fabrication}

To fabricate the printable composites, PCL (M.W. = 43,000-50,000; Polysciences, Warrington, PA, USA) was molten at $90^{\circ} \mathrm{C}$ for 10 minutes. After which, MCS or MCSF powder suspended in ethanol was slowly dropped into the molten PCL until it reached a final content of $50 \mathrm{wt} \%$. The mixtures were dried in a $100{ }^{\circ} \mathrm{C}$ oven for $24 \mathrm{~h}$. Finally, there were three concentrations of FGF-2 in the scaffolds that coded MCS, MCSF5, and MCSF10. For the fabrication of composite scaffolds, the composite pastes were loaded into syringes and dispensed through a $80^{\circ} \mathrm{C}$ steel nozzle with an inner diameter of $400 \mu \mathrm{m}$ by applying pneumatic pressures of $200-400 \mathrm{kPa}$ at printing speeds of $1.5-2 \mathrm{~mm} / \mathrm{s}$. The designs and manufacture of 3D-printed scaffolds used the Bio-Scaffolder 3.1 system (GeSiM, Grosserkmannsdorf, Germany). The thickness of each layer in scaffolds was controlled to $300 \mu \mathrm{m}$ and the dimension of the scaffolds were $6 \mathrm{~mm} \times 6 \mathrm{~mm} \times 10 \mathrm{~mm}$ rectangle. X-ray diffractometry (XRD) was used to analyze the crystallization properties and phase components of the MCS and MCSF scaffolds.

\subsection{FGF-2 Release and Bioactivity}

The FGF-2 release profiles of MCSF5 and MCSF10 scaffolds were measured by immersing the scaffolds in the Dulbecco's modified Eagle's medium (DMEM, Invitrogen) with 1\% penicillin/ streptomycin at $37^{\circ}$ for a period of 14 days. To mimic the release condition of FGF-2 during in vitro experiments, DMEM was employed as the solution for the drug release experiment, and separated scaffolds were analyzed at each time point. The amount of FGF-2 released from different scaffolds was quantitatively measured using ELISA kit (Invitrogen) according to the manufacturer's instructions and the results were obtained in triplicate from three independent experiments. Additionally, a field-emission scanning electron microscopy (FE-SEM, JEOL) at a low secondary electron image (LEI) mode and $3 \mathrm{kV}$ acceleration voltage were utilized to visualize the surface morphologies of the 3D-printed scaffolds before and after incubation in DMEM within a period of 7 days. The formation apatite precipitate on different scaffold surfaces during FGF-2 release was assessed by XRD.

\subsection{Cell Proliferation}

Human Wharton's jelly mesenchymal stem cells (hWJMSCs) used in this study were obtained from Bioresource Collection and Research Center (Hsin-Chu, Taiwan). The cells were maintained in the commercially available mesenchymal stem cell medium (\#7501, Sciencell, Carlsbad, CA, USA) and cells at passage between 3-7 were used in this study. Prior to cell experiments, all scaffolds were sterilized by soaking them in $75 \%$ alcohol followed by exposure to UV irradiation for $30 \mathrm{~min}$. To determine the cytotoxicity of different scaffolds, the cell suspensions at a density of $5 \times 10^{4}$ cells $/ \mathrm{mL}$ were seeded on each scaffold and cultured for various periods. At each culture period, all scaffolds were transferred to a new culture plate and washed with cold phosphate-buffered saline (PBS, Invitrogen) three times. Then, $1 \mathrm{~mL}$ of culture medium containing $0.5 \mathrm{mg} / \mathrm{mL}$ of 3-(4,5-dimethylthiazol-2-yl)-2,5-diphenyltetrazolium bromide (MTT, Sigma-Aldrich) was added and incubated at $37^{\circ} \mathrm{C}$ for $4 \mathrm{~h}$. The purple colored formazan crystal was then dissolved with dimethylsulfoxide (DMSO, Sigma-Aldrich), and the absorbance at $570 \mathrm{~nm}$ with a reference wavelength of $650 \mathrm{~nm}$ was recorded using a microplate reader (TECAN Infinite Pro M200, Männedorf, Switzerland). The absorbance values were obtained in triplicate from three independent experiments. The cells cultured on tissue cultured plates were used as control groups $(\mathrm{Ctl})$ for the in vitro experiments.

\subsection{Western Blot}

After 1 day of incubation, hWJMSCs cultured on the scaffolds were lysed by using a NP40 buffer solution (Invitrogen) and the level of protein concentrations in different lysates was determined by bicinchoninic acid (BCA) protein assay kit (Invitrogen). Sodium dodecyl sulfate-polyacrylamide -polyacrylamide gel electrophoresis (SDS-PAGE) was used to segregate the cell lysates (40 $\mu \mathrm{g}$ protein) and the segregated proteins were transferred onto membranes (Merck Millipore, Billerica, MA, USA). 
Blocking treatment was performed by immersing the membrane in a PBS solution containing $4 \%$ bovine serum albumin (BSA, Invitrogen) and 0.1\% tween-20 detergent (Sigma-Aldrich) for $1 \mathrm{~h}$ before exposing them to primary antibodies against FGF receptor (FGFR, Thermo Fisher, Waltham, MA, USA) and $\beta$-actin (Abcam, Boston, MA, USA) for $2 \mathrm{~h}$ for further immunoblotting. Afterward, horseradish peroxidase(HRP)-conjugated secondary antibodies (Santa Cruz Biotechnology, Santa Cruz, CA, USA) were incubated with the PVDF membranes for another $1 \mathrm{~h}$ for further visualization using chemiluminescence. The results of FGFR expression were normalized to $\beta$-actin and the semi-quantitative results were obtained in triplicate from three independent experiments.

\subsection{ALP Activity}

Alkaline phosphatase (ALP) is an osteogenesis-related biomarker that can be used to evaluate the early-stage osteogenic differentiation level of cells cultured on the scaffolds. Briefly, hWJMSCs were cultured on different scaffolds in the osteogenic medium (StemPro ${ }^{\mathrm{TM}}$ osteogenesis differentiation kit, Invitrogen) for 3, 7, and 14 days. At the end of each cultured period, the cells were washed with cold saline solution $(0.9 \% \mathrm{NaCl})$ and then lysed in $0.2 \% \mathrm{NP}-40$. The lysates were centrifuged at $2000 \mathrm{rpm}$ for $15 \mathrm{~min}$. Subsequently, the supernatants were incubated with $p$-nitrophenyl phosphate ( $p$ NPP, Sigma) solution at $37^{\circ} \mathrm{C}$ for $1 \mathrm{~h}$. Then, $5 \mathrm{~N} \mathrm{NaOH}$ was used to terminate the above reactions and the absorbance at a wavelength of $405 \mathrm{~nm}$ of the mixture solutions was read by using a microplate reader and the absorbance values were obtained in triplicate from three independent experiments. All data were normalized to the concentration of total protein obtained from BCA protein assay kit. The cells cultured on tissue culture plates were used as controls $(\mathrm{Ctl})$ for this study.

\subsection{Rabbit Model of Femoral Bone Defects}

The in vivo experimental protocols used in this study were approved by the Animal Experimental Ethics Committee of Chung Shan Medical University. New Zealand white male rabbits with an age of 3 months and a weight between $1.8-2.0 \mathrm{~kg}$ were used in this study. The animals were randomly divided into three groups, namely MCS, MCSF5, and MCSF10. After being anesthetized by $5 \%$ isoflurane supplied with $100 \%$ oxygen by using a gas anesthesia machine (Engler ADS1000, Hialeah, FL, USA), the hair on the hind legs was shaved. Subsequently, alcohol and iodine were used for disinfection before dissection of skin. The muscle fascia was dissected carefully to expose the femur without causing adverse injury to nerves and blood vessels. Critical-sized femur defects with a diameter of $6 \mathrm{~mm}$ and a depth of $6 \mathrm{~mm}$ were created by using a low-speed bone drill. The scaffold that was sterilized by soaking it in $75 \%$ ethanol for $1 \mathrm{~h}$ was then implanted into the defect site. Finally, the rabbits received suture closure and anti-inflammatory ointment was administrated on the wound surface. At 4 weeks post-implantation, the rabbits were sacrificed with $\mathrm{CO}_{2}$ asphyxiation. The scaffolds and bone tissue were harvested and subsequently fixed in $10 \%$ normal formalin.

\subsection{Microcomputed Tomography}

The microcomputed tomographic images of the specimens were obtained by using a high spatial resolution microcomputed tomography system ( $\mu \mathrm{CT}$, SkyScan 1076, SkyScan Inc., Kontich, Belgium) equipped with a 1.4 M X-ray CCD camera at a tube voltage and current of $70 \mathrm{~V}$ and $70 \mathrm{~A}$, respectively. The images of the femur scans were acquired at a maximum tube current of $0.2 \mathrm{~mA}$ and a maximum tube voltage of $160 \mathrm{kV}$ with a $1 \mathrm{~mm}$ focus size. A total of 400 images for the slice increment of $30 \mathrm{~mm}$ was taken. The 3D model of the femur scans was created by using 3Di-Cat thresholding for further assessment and quantitative histomorphometric analysis. The neo-bony tissues, the bone volume per tissue volume (BV/TV), and trabecular thickness (Tb.Th) in bone specimens were recognized by using a SkyScan software. The results of BV/TV and Tb.Th were obtained in triplicate from three independent experiments. 


\subsection{Histological Staining}

After being fixed in $10 \%$ normal formalin for 2 days, the specimens were rinsed and embedded in an optimum cutting temperature compound (OCT®, KMA-0100-00A, CellPath Ltd., Newtown, Wales, UK) and subsequently sectioned into transparent slices with a thickness of $6 \mu \mathrm{m}$ along with the longitudinal direction of the implanted scaffolds by using a sawing microtome technique. The sections were stained with hematoxylin and eosin (HE), Masson's trichrome stain kit (MT, ScyTek Lab, West Logan, UT, USA) or the von Kossa kit (VK, ScyTek Lab) according to manufacturers' instructions. Histological images of the HE-, MT-, and VK-stained sections were visualized by using a BX53 Olympus microscope.

\subsection{Statistical Analyses}

The one-way analysis of variance (ANOVA) was used to determine the existence of statistical differences between means of every quantitative result. The Scheffé's test was used to account for multiple comparisons amongst group means. All data were considered to be statistically significant with a p-value less than 0.05 .

\section{Results and Discussion}

\subsection{Characterization of the MCSF/PCL Scaffold}

In the present study, the CTAB-templated approach was employed to fabricate calcium silicate nanoparticles with a mesoporous structure for serving as cargoes of FGF-2. Figure 1A reveals the TEM micrograph of MCS nanoparticles. It can be observed that MCS with a size of $229.8 \pm 34.9 \mathrm{~nm}$ exhibited an ordered interior mesoporous structure with a pore size of $2.3 \pm 0.4 \mathrm{~nm}$, which was in accordance with previous results $[27,30]$. In this study, we utilized the extrusion-based 3D printer to fabricate a scaffold used for bone tissue engineering due to its advances in scalable and on-demand designing/manufacturing of constructs with complex shapes and structures. Unfortunately, the osteo/odontogenic activity of PCL is poor. To optimize the biological performance of the 3D printed scaffold, the osteoconductive MCS fillers loaded with FGF-2 were blended into PCL for enhancing the osteogenicity of the scaffold. To this end, the concentration of the fillers should be as high as possible. Therefore, PCL composites containing $50 \mathrm{wt} \%$ of MCS and MCSF were used in the study due to the fact that the composite with a filler content of $50 \mathrm{wt} \%$ exhibits acceptable printability. The viscosity of the composite would remarkably increase while the filler content reached $60 \mathrm{wt} \%$, resulting in the loss of printability and structural fidelity of the scaffolds. After introducing MCS nanoparticles into PCL, the open-porous composite scaffold was able to be fabricated through successful deposition and layer-by-layer fusion of molten PCL/MCS struts with a diameter of $500 \mu \mathrm{m}$ in a laydown pattern of $0^{\circ} / 90^{\circ}$. Although the pore size of the scaffolds along with $z$-axis direction can be controlled by adjusting the distance between the deposited struts, smaller side-wall pores were usually obtained due to the occurrence of the plastic collapse of the as-deposited struts [31].

According to previous findings, porous scaffolds with a pore size of up to $300 \mu \mathrm{m}$ were sufficient to support the ingrowth of neo-bony tissues [32]. However, some studies have suggested that scaffolds with larger pores would be considered suitable for guiding bone healing because of the rapid occupation of the peripheral pores by fibrous tissues, which impede the infiltration of neo-vessels and bony tissues into scaffolds with smaller pore sizes [33]. Considering the efficiency of neo-tissue penetration and the nutrient/waste transfer could be jeopardized due to the diminished size of side-wall pores, a double layer printed configuration was used to manufacture 3D scaffolds with a wall pore size of about $600 \mu \mathrm{m}$ (Figure 1B) [34]. The XRD results (as shown in Figure 1C) of both MCS and MCSF show two typical characteristic peaks located at $2 \theta$ of $21.3^{\circ}$ and $23.6^{\circ}$, which could be attributed to the formation of orthorhombic crystalline structure of PCL [35]. This result implies that the MCS composite retained the thermoplasticity of PCL and consequently allowed the molten pastes to be solidified through temperature-controlled crystallization. Peaks showed at $29.6^{\circ}$ and in a $2 \theta$ range between $32^{\circ}$ to $34^{\circ}$, 
indicating that the major phase composition of the inorganic component incorporated in the composite were calcium silicate hydrate $(\mathrm{CSH})$ and $\beta$-dicalcium silicate $\left(\beta-\mathrm{Ca}_{2} \mathrm{SiO}_{4}\right)$, respectively [36].
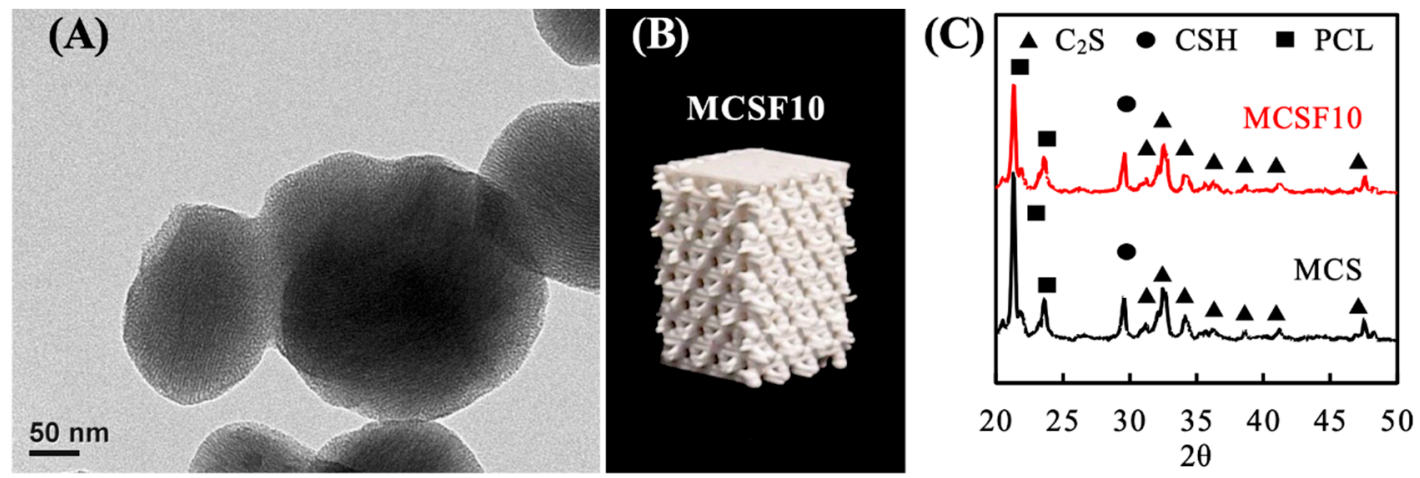

Figure 1. (A) TEM micrograph of calcium silicate-based mesoporous nanoparticles (MCS). (B) Photographs of the polycaprolactone (PCL)/MCSF10 scaffold fabricated through deposition of the molten struts layer-by-layer in a laydown pattern of $0^{\circ} / 90^{\circ}$. (C) XRD patterns of MCS- and MCSF10-incorporated PCL composites.

First, the measured loading capacities of MCSF5 and MCSF10 powders were 742 and 1219 pg per $1 \mathrm{~g}$ of MCS powder, respectively. The release profiles of FGF-2 from the MCSF scaffolds (as shown in Figure 2) indicate that the burst release was noticed during the first three days of which about $9 \%$ (15 pg) and 12\% (41 pg) of the loaded FGF-2 was released from MCSF5 and MCSF10, respectively. Afterwards, the release rate would slightly reduce in both groups and the release amounts of FGF-2 from MCSF5 and MCSF10 were increased to approximately 18\% (32 pg) and 25\% (90 pg) at incubation period of 14 days, respectively. In the present study, the MCSF nanoparticles were blended in PCL, implying that PCL can block the exposure of MCSF, especially for that located in the interior of the scaffolds, to the solution. Thus, the fast release of FGF-2 in the early stage could be contributed by the MCSF located in the superficial zone of the scaffolds. Although the extremely slow degradation rate of PCL could completely impede the release of FGF-2 from the scaffolds, our results indicate that sustained release of FGF-2 from the scaffolds was achieved in an experimental period of 14 days due to the possible reason that the lack of covalent interactions between the fillers and PCL matrices might allow diffusion of solution into the scaffold and subsequently interacted with FGF-2-loaded nanoparticles to support the sustained release of FGF-2 from the scaffolds. It could be noticed that the MCSF10 shows a faster release rate than MCSF5. This was probably attributed to the higher amount of FGF-2 that could be loaded into the MCS nanoparticles while the MCS was incubated with a high concentration of FGF-2 solution [37]. The loaded FGF-2 was capable of gradually releasing from the scaffolds due to the fact that the MCSF was buried in the PCL matrix, and subsequently delayed it from contact with the solution. In general, PCL was not considered as an ideal hydrophilic drug loading matrix due to its hydrophobic nature and extremely slow degradation rate [38]. In our case, FGF-2 was loaded in the hydrophilic MCS and then blended into the PCL matrix, which favorited the penetration of water into the interface between the MCS fillers and PCL matrix to consequently support a sustained release of FGF-2 from the scaffolds [35].

\subsection{In Vitro Soaking}

The in vitro bioactivity of the 3D printed-MCS and MCSF scaffolds was assessed by examining changes in terms of surface morphology and phase composition of scaffolds before and after immersion in DMEM within seven days. Figure 3 reveals the evolution of surface morphologies of MCS, MCSF5, and MCSF10 as a function of soaking period. SEM micrographs demonstrate that the as-prepared scaffolds exhibited rough surface features, which can be a result of the formation of spherulite semi-crystalline of PCL [39]. Exposed fillers were barely found on the surface of the struts because 
of the rheological characteristics of molten PCL [40]. After being immersed in DMEM for one day, all surfaces were covered by a layer of nano-sized precipitates. A growing number of the precipitated particles were formed, and those particles subsequently fused to each other to develop a dense precipitate layer on all scaffolds at an incubation period of three days. The size of the precipitates and the thickness of the precipitate layer would remarkably increase while the DMEM immersion period was extended to seven days [41]. The results of XRD spectra (Figure 4) indicate that the phase compositions of MCS and MCSF after being immersed in DMEM for one and three days were identical to that of as-prepared scaffolds. However, a new peak located at $2 \theta$ of $25.9^{\circ}$ along with broad and diffused peaks at $2 \theta$ between $31.6^{\circ}$ to $32.8^{\circ}$, which could be assigned to apatite, emerged while immersing the scaffolds in DMEM for seven days [11]. Moreover, the intensities of characteristic peaks corresponding to $\mathrm{PCL}$ and $\beta-\mathrm{Ca}_{2} \mathrm{SiO}_{4}$ gradually decreased with increasing immersion period. These results indicate that the precipitated layer observed by SEM could be attributed to the formation of apatite [42].

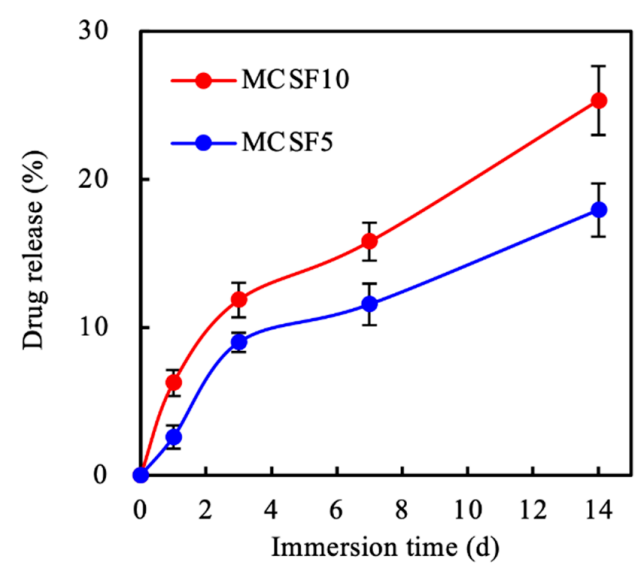

Figure 2. Evaluation of basic fibroblast growth factor (FGF-2) released from MCSF5 and MCSF10 scaffolds soaked in PBS at $37^{\circ} \mathrm{C}$ as a function of time.

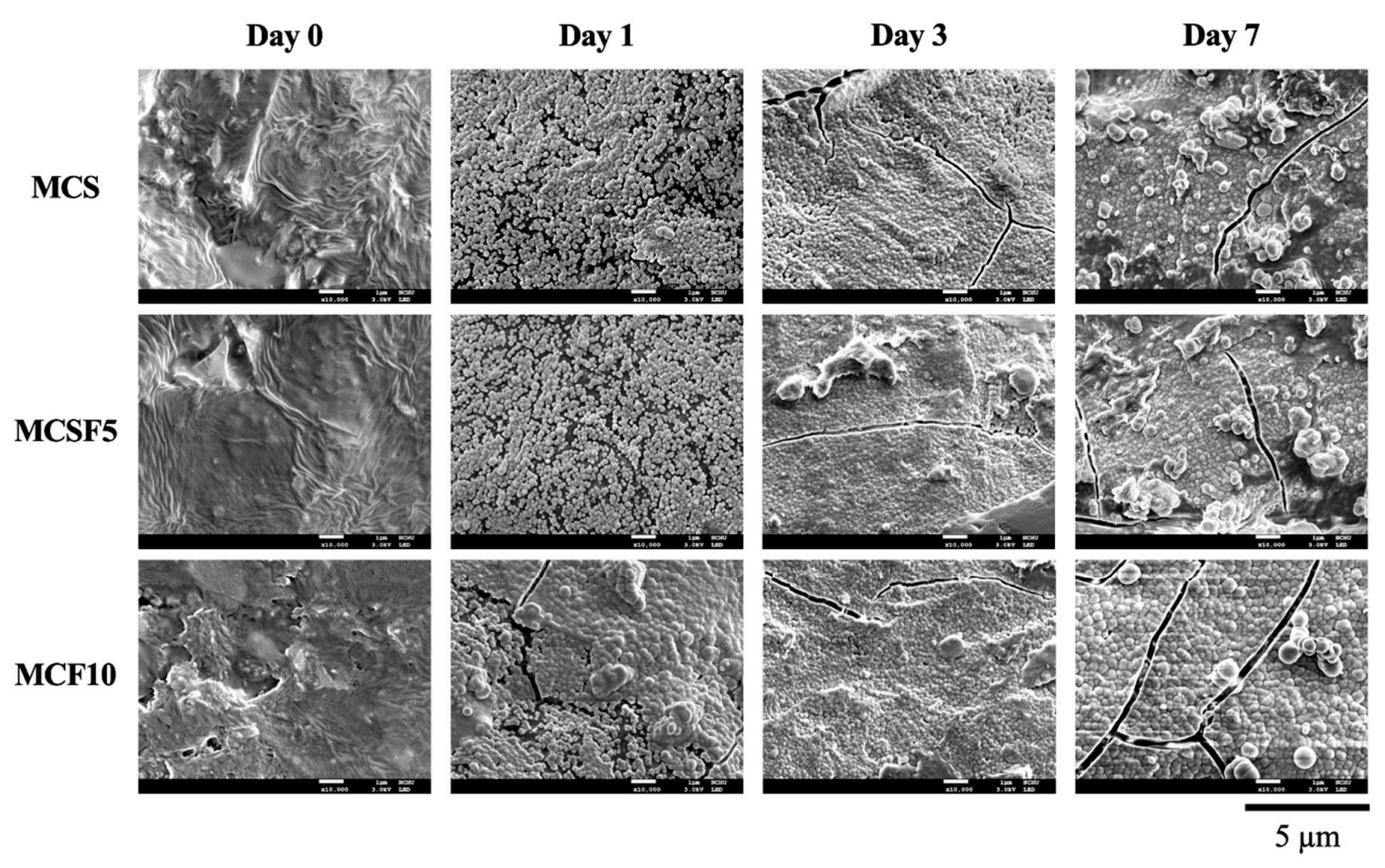

Figure 3. SEM micrographs of MCS, MCSF5, and MCSF10 scaffolds before and after immersion in Dulbecco's modified Eagle's medium (DMEM) at $37^{\circ} \mathrm{C}$ for 1 and 7 days. 
Day 1

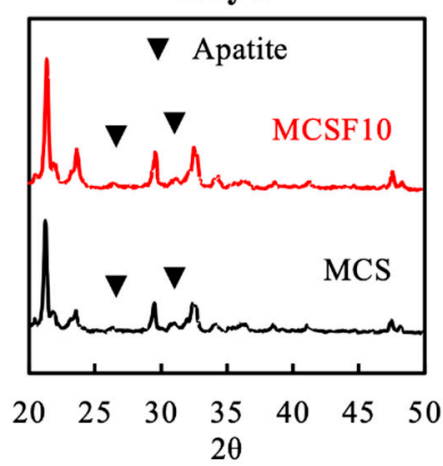

Day 3

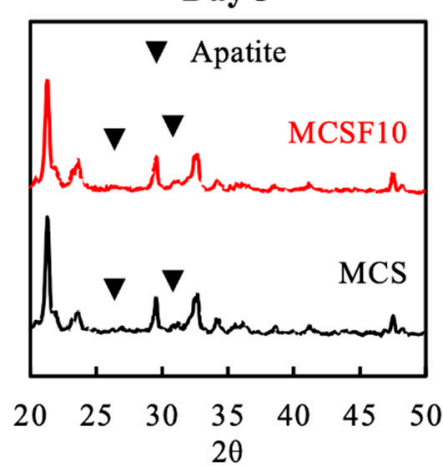

Day 7

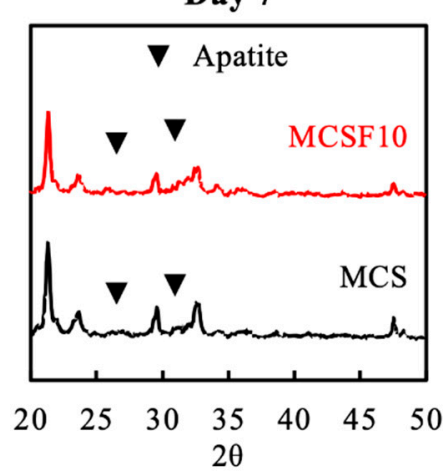

Figure 4. XRD patterns of MCS and MCSF10 scaffolds after immersion in DMEM at $37^{\circ} \mathrm{C}$ for 1,3 , and 7 days.

\subsection{In Vitro Experiments}

Previous studies have suggested that the FGF signaling pathway activated by association with FGFs and FGFRs plays a critical role in manipulating proliferation and osteogenesis differentiation of osteogenic progenitor cells. However, it had been documented that FGF-2 can exert both activatory and inhibitory effects on the proliferation and early-stage differentiation of osteogenic progenitor cells depending on the in situ concentration of FGF-2, in which the upregulation effect of exogenous FGF-2 on cells can be diminished while a high dose is administrated [43]. To verify the effect of incorporated FGF-2/MCS within PCL on growth of hWJMSCs, proliferation of cells cultured on different scaffolds were examined. The PrestoBlue assay was used to analyze proliferation of hWJMSCs cultured on MCS and MCSF scaffolds quantitatively and is shown in Figure 5. It indicated that the amounts of cells either on the tissue culture plate (Ctl) or composite scaffolds (MCS, MCSF5, and MCSF10) progressively increased within culturing time, and each group indicated statistical differences at every time interval $(p<0.05)$. Among these groups, $\mathrm{Ctl}$ and MCSF10 revealed the lowest and highest absorbances at all culture periods, respectively. Approximately 1.5- and 1.3-fold increases in the absorbance values of MCSF10 group were detected in comparison with that of Ctl and MCS, respectively, at a culture period of up to seven days. In contrast to Ctl, the MCS scaffolds showed comparable levels of absorbance values at each culture period, and a significant difference was detected between cells cultured on $\mathrm{Ctl}$ and MCS for 14 days of culturing $(p<0.05)$. Both MCSF5 and MCSF10 groups showed significantly higher amounts of cells than Ctl and MCS groups for a culture period of up to seven days $(p<0.05)$, demonstrating that the incorporation of FGF-2 in the scaffold would be beneficial to enhance the proliferation of cells. Interestingly, the FGF-2-induced dose-dependent increase in cell proliferation of hWJMSCs was not found. The absorbance levels of cells cultured on MCSF5 and MCSF10 stayed similar in a culture period of seven days despite the cells being exposed to a larger amount of FGF-2 while being cultured on MCSF10 due to the higher loading capacity and faster release kinetics. In fact, evidence has suggested that depression in cell growth could occur when exposing cells to a high dose of FGF-2. However, the high dose FGF-2-induced growth inhibition was not found in the present study, implying that the local concentration of the released FGF-2 from MCSF5 and MCSF10 might be in a range that is beneficial for enhancing cell proliferation [44].

To further assess the effect of the incorporation of FGF-2 in MCS scaffold on osteogenesis differentiation of hWJMSCs, FGFR expression and ALP activity of cells cultured on different scaffolds were examined. Figure 6 reveals the FGFR expression levels of cells cultured on different scaffolds. The semi-quantitative result of Western blot analysis illustrated that expression of FGFR of cells could be remarkably upregulated in the presence of FGF-2 released from the scaffolds. Significant differences can be found between every group among all scaffolds $(p<0.05)$. The FGFR levels of cells cultured on MCSF5 and MCSF10 were 1.6- and 2.2-fold higher than that on MCS, respectively. Moreover, our results indicate that the up-regulatory effect of FGF-2 on FGFR expression was in a 
dose-dependent manner. The extensive study demonstrated FGF-2 was an important mitogen that could manipulate proliferation and differentiation behaviors of mesenchymal stem cells and further regulated downstream osteogenic-related proteins via the FGF/FGFR/MAPK signaling pathway [45]. The result of ALP activity (Figure 7) demonstrated a similar trend with that of cell proliferation and FGFR expression; however, the ALP activity of cells cultured on MCS scaffold for seven and 14 days was about 1.3-fold higher than that on Ctl with significant differences $(p<0.05)$, demonstrating the effectiveness of MCS nanoparticles on modulating the early-stage differentiation of hWJMSCs into osteogenic lineages as indicated by our previous results [37]. It is worth noting that cells cultured on MCSF10 revealed a significantly higher level of ALP activity than that of others at culture periods of seven and 14 days $(p<0.05)$.

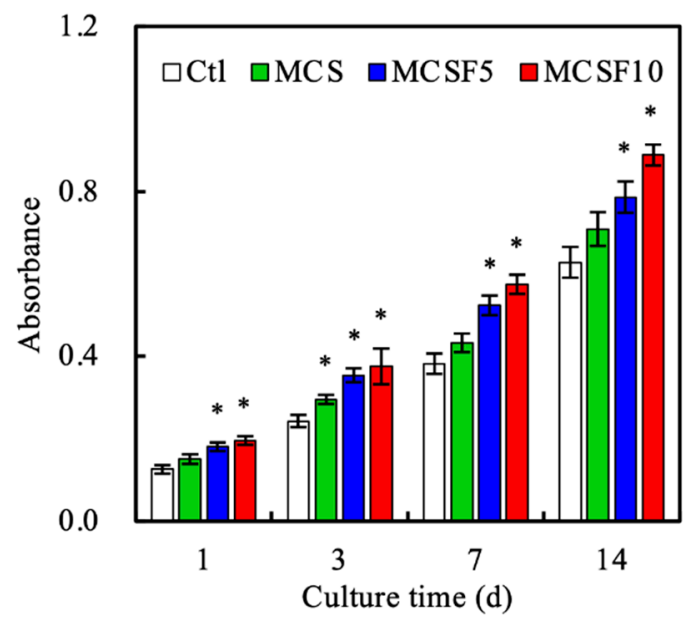

Figure 5. Proliferation of human Wharton's jelly mesenchymal stem cells (hWJMSCs) cultured on MCS, MCSF5, and MCSF10. * indicates significant difference $(p<0.05)$ from control $(\mathrm{Ctl})$ group.

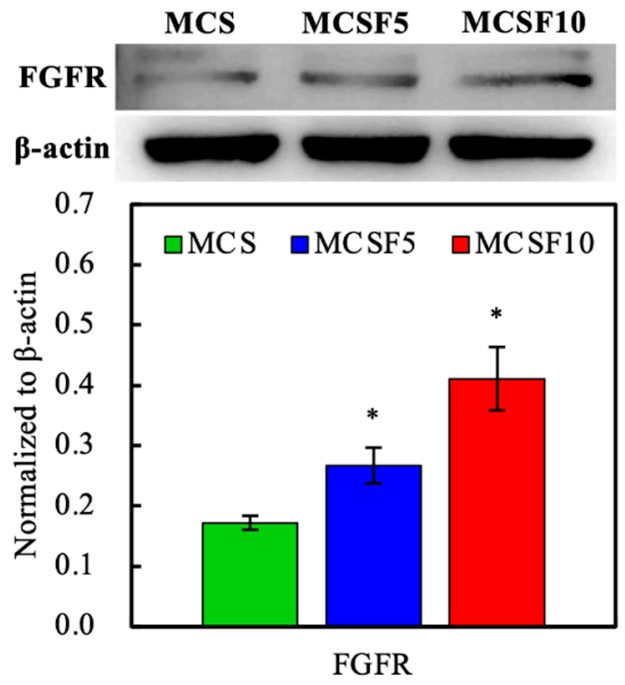

Figure 6. The fibroblast growth factor receptor (FGFR) expression of hWJMSCs cultured on different scaffolds. ${ }^{*}$ indicates significant difference $(p<0.05)$ from MCS group. 


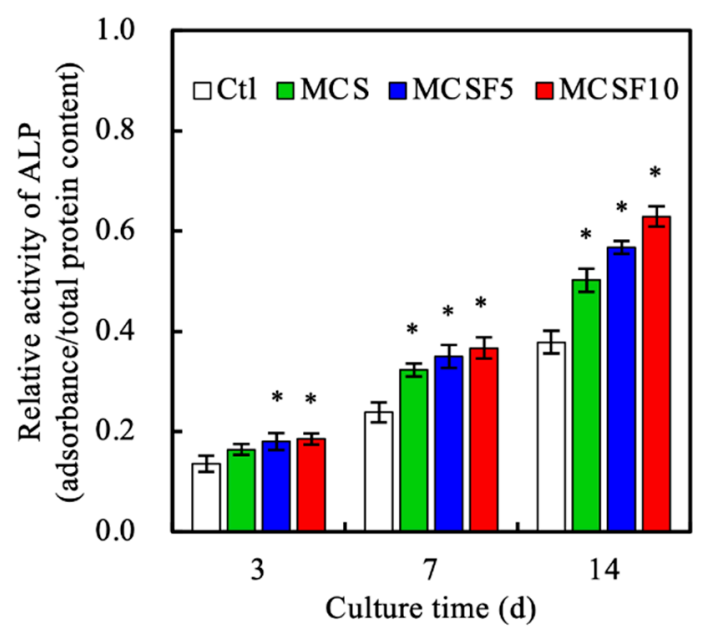

Figure 7. The alkaline phosphatase (ALP) activity of hWJMSCs cultured on different scaffolds for 3, 7, and 14 days. ${ }^{*}$ indicates significant difference $(p<0.05)$ from $\mathrm{Ctl}$ group.

\subsection{In Vivo Experiments}

The in vivo osteogenesis capacities of MCS scaffolds in the absence or presence of FGF-2 were investigated by a rabbit femur model. As seen in Figure 8A, newly formed bone tissues that penetrated into the interior of scaffolds can be observed in all scaffolds after four weeks post-implantation, demonstrating that the double layer printed configuration of the scaffolds possessed a superior size of side-wall pores that allowed rapid penetration of neo-bone tissues into the pores of scaffolds within four weeks [46]. Noticeably, MCSF scaffolds revealed better bone repair efficiency when compared to bare MCS scaffolds. It could be found in $\mu \mathrm{CT}$ images that the pores of MCSF scaffolds were almost fully occupied by dense neo-bone tissues whereas neo-bone tissues with a loose structure could be only observed in the peripheral site of MCS scaffolds [46]. The morphometrical analysis (Figure 8B) revealed significantly higher values $(p<0.05)$ in the BV/TV ratio of MCSF groups $(27.9 \%$ and $33.2 \%$ for MCSF5 and MCSF10, respectively) in contrast to MCS one (22.8\%) after been implanted for four weeks. The results of $\mathrm{Tb}$.Th (Figure $8 \mathrm{C}$ ) demonstrate a similar trend to that observed in the BV/TV ratio. The Tb.Th values of MCS, MCSF5, and MCSF10 were $0.109,0.147$, and $0.188 \mathrm{~mm}$, respectively, which increased with increasing amount of FGF-2. Significant between-group differences were found in both BV/TV ratio and Tb.Th measurements $(p<0.05)$, implying that bone healing efficiency of the scaffolds would be determined by the amount of loaded FGF-2. These results were in good accordance with the results of ALP activity. Prins et al. suggested that the level of ALP activity could be employed as an indicator for the prediction of in vivo bone forming capability of isolated bone marrow-derived MSCs and their findings demonstrated that MCSs-induced osteogenicity could only be achieved on the cells receiving ALP induction treatments [47]. Despite the positive effects of MCS and MCSF scaffolds on the osteogenesis in the rabbit femur defect model that were obtained, detailed in vitro analysis used for quantitative and qualitative measurements of osteogenic biomarkers, such as ALP, osteopontin, and osteocalcin, should be conducted to better elucidate the possible underlying mechanisms of the MCS and MCSF composite scaffolds-guided regeneration of bone tissues. 

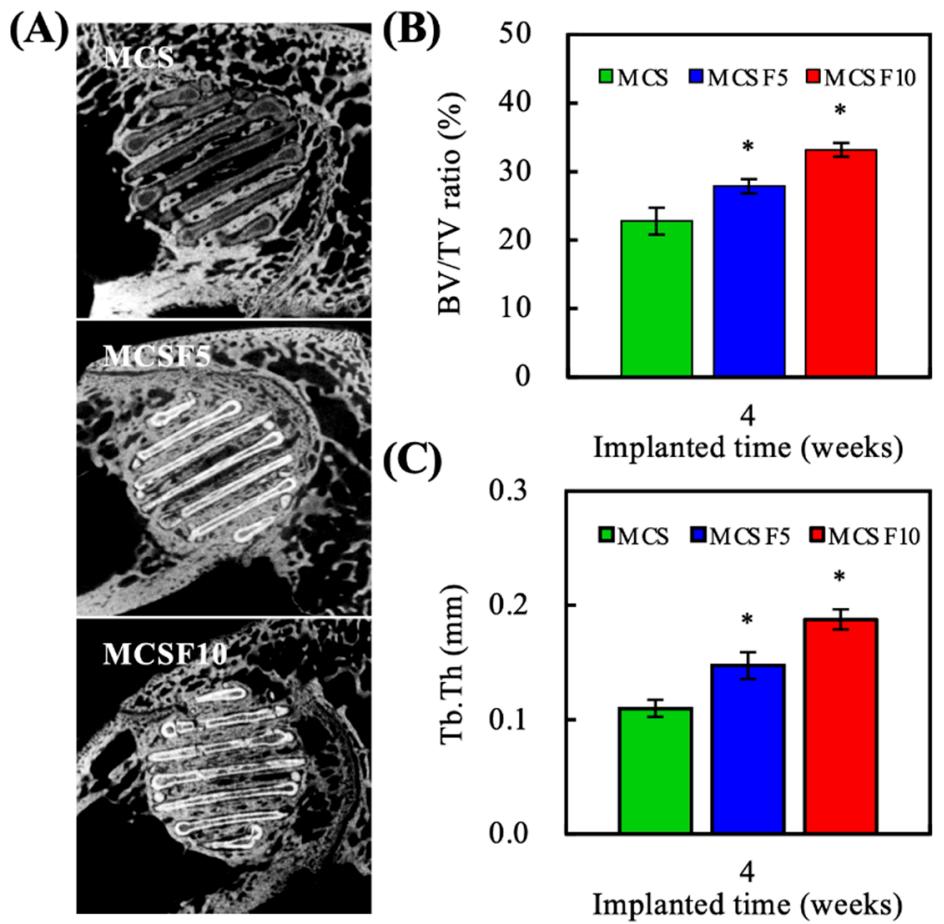

Figure 8. (A) The $\mu \mathrm{CT}$ images, (B) data analysis of relative bone mass volume (BV/TV), and (C) quantitative measurement of trabecular thickness $(\mathrm{Tb} . \mathrm{Th})$ of the femur defects after implantation with different 3D printed scaffolds for 4 weeks. ${ }^{*}$ indicates significant difference $(p<0.05)$ from MCS group.

To further assess the osteogenic activity of different scaffolds, hematoxylin and eosin (HE) stain, Masson's trichrome (MT) stain, and von Kossa (VK) stain were conducted and the results are shown in Figure 9. The HE staining images indicate that no apparent inflammatory cells were found in all scaffolds, demonstrating the good biocompatibility of the PCL/MCS composite in the absence and presence of FGF-2. It could be observed that the space between struts of scaffolds was completely filled with neo-tissues, which were mainly composed of dense collagen matric (purple) and immature osteoids (light-pink). In contrast to MCSF10, a noticeable amount of osteoids that covered the surface of struts could be obtained in MCS and MCSF5. In the MT staining images, the deep blue color depicts the formation of mineralized matric. It is of note that the amount of calcified collagen matric increased with increasing quantity of FGF-2 in the scaffolds, which can be validated by the presence of dark brown to black colored tissues showed in the VK staining images of undecalcified specimens. Xia and coworkers [48] demonstrated a comparison study of BMP-2-loaded PCL and PCL/hydroxyapatite (HA) scaffolds fabricated by a selective laser sintering (SLS) technique on the in vivo regeneration of bone tissues in a rabbit femur defect model with defect diameter of $6 \mathrm{~mm}$ and depth of $10 \mathrm{~mm}$. Their results suggest that new bone formation was barely observed in the non-treated defects at nine weeks post-surgery unless BMP-2-loaded PCL or PCL/HA scaffolds were implanted in the defect site. Other studies also demonstrate similar results. For example, Liu et al. [16] reported that impaired regeneration of bony tissues was observed in Sprague Dawley rat skull defects without implantation of 3D-printed PCL or strontium (Sr)-doped HA/PCL scaffolds at 12 weeks post-surgery. Additionally, the defects could be occupied by fibrous tissues. In contrast, the bone healing efficiency of the defects was improved by introducing the scaffolds to the defected site and the formation of neo-bony tissues were remarkably increased if osteogenic elements (HA and SrHA) were present in the scaffolds, indicating the crucial roles of osteoconductive/inductive dopant in modulating osteogenicity of 3D-printed PCL scaffolds. Despite our results demonstrating that MCSF may possess appealing osteogenicity for guiding the regeneration and ingrowth of neo-bone, the level of vascularization of the neo-tissue should be taken into account since insufficient vascularization can result in impaired exchanges of nutrition/oxygen and diminish the penetration depth of neo-bone 
into scaffolds. Additionally, cross-talks between vascular endothelial cells and osteoprogenitor cells based on the paracrine secretion of various growth factors and cell-cell communications have been considered as crucial processes involved in bone tissue regeneration [49]. In our results, the formation of vascular lumens (as indicated by red arrows) was achieved in MCSF scaffolds, implying that an elevated osteogenesis might possibly result from the in vivo effectiveness of FGF-2 in modulating the osteogenic differentiation and angiogenic activity of the corresponding progenitor cells [25]. Together, these results demonstrate that MCS can not only serve as functional fillers to promote the bioactivity and osteoconductivity of the 3D-printed PCL scaffolds, but also as loading cargoes of therapeutic agents to achieve sustained release of FGF-2 from the composite scaffolds and consequently facilitate in vivo regeneration of bone defects.

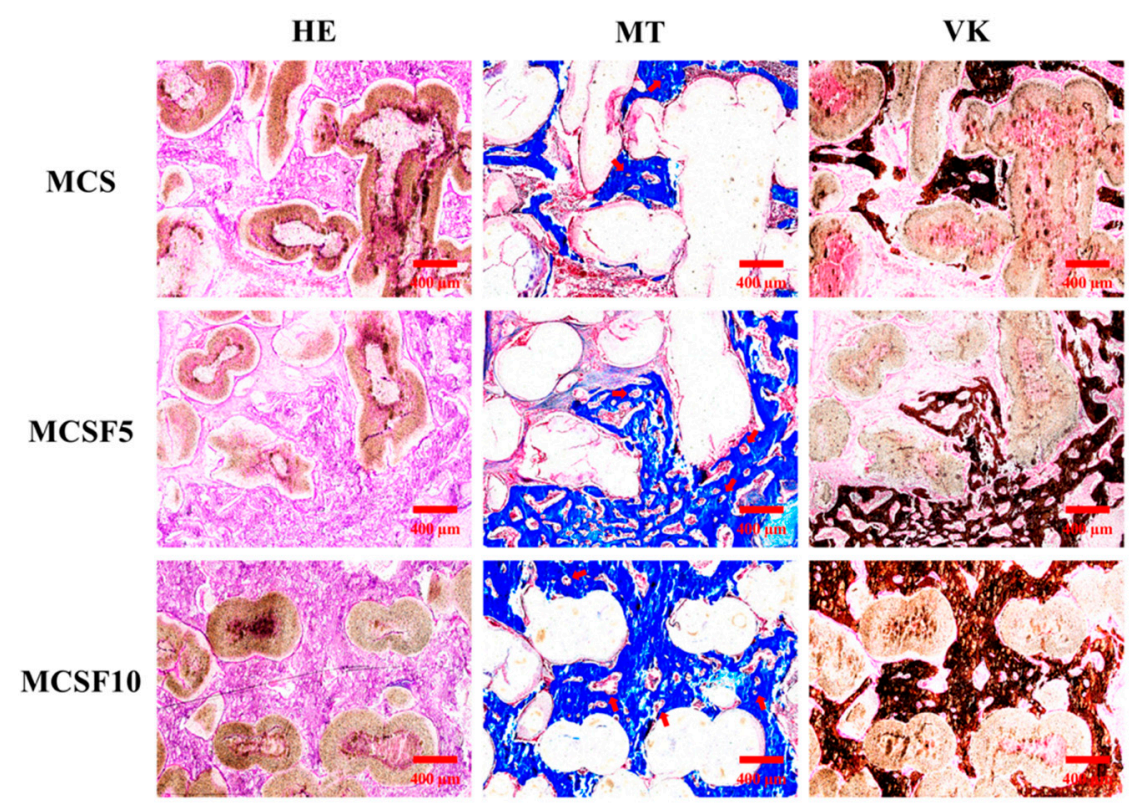

Figure 9. Hematoxylin and eosin (HE), Masson's trichrome, and von Kossa staining of the femur defects after implantation with different 3D printed scaffolds for 4 weeks. Red arrows indicate the neovascular lumens.

\section{Conclusions}

In the present study, CS nanoparticles with a particle size of about $230 \mathrm{~nm}$ and a wellaligned mesoporous structure were successfully synthesized through the CTAB-templated method. The existence of the mesopores impregnated the FGF-2 loading in MCS powders. The composites of PCL contained $50 \mathrm{wt} \% \mathrm{MCS}$ and MCSF and were 3D printable, which enables the precise control of the macro-structure of the scaffolds and the MCSF scaffolds possessed the ability to sustainedly release FGF-2 loaded in the fillers. Furthermore, the MCS and MCSF composite scaffolds retained excellent in vitro apatite-forming ability of CS, which may be beneficial for adhesion, proliferation, and early-stage differentiation of osteogenic progenitor cells. More importantly, the presence of FGF-2 in the scaffolds could support the proliferation and upregulated the ALP activity of hWJMSCs. In vivo results demonstrate that the healing efficiency of femur bone defects in rabbits was significantly enhanced while incorporating FGF-2 in the composite scaffolds. Therefore, we believe the proposed approach for fabrication of 3D printed MCSF/PCL could be considered as a potential bone graft for bone tissue regeneration.

Author Contributions: Data curation, C.-T.K.; formal analysis, T.-T.H.; funding acquisition, Y.-J.C.; investigation, Y.-H.L. and T.-H.H.; methodology, Y.-J.C. and T.-H.H.; writing—original draft, C.-T.K. and T.-T.H.; writing一review and editing, C.-C.H. All authors have read and agreed to the published version of the manuscript. 
Funding: The authors acknowledge the receipt of grants from the Ministry of Science and Technology (MOST 106-2314-B-040-003-MY3) of Taiwan, the China Medical University Hospital (DMR-109-108) of Taiwan, and the Chung Shan Medical University Hospital (CSH-2019-C-037).

Acknowledgments: Experiments and data analysis were performed in part through the use of the Medical Research Core Facilities Center, Office of Research and Development at the China Medical University. SEM image was assisted by Mrs. Ya-Hsun Lin (MOST 108-2731-M-005-001).

Conflicts of Interest: The authors declare no conflict of interest.

\section{References}

1. Roopavath, U.K.; Malferrari, S.; Van Haver, A.; Verstreken, F.; Rath, S.N.; Kalaskar, D.M. Optimization of extrusion based ceramic 3D printing process for complex bony designs. Mater. Design 2019, 162, $263-270$. [CrossRef]

2. Bian, T.; Zhao, K.; Meng, Q.; Tang, Y.; Jiao, H.; Luo, J. The construction and performance of multi-level hierarchical hydroxyapatite (HA)/collagen composite implant based on biomimetic bone Haversian motif. Mater. Design 2019, 162, 60-69. [CrossRef]

3. Vijayavenkataraman, S.; Kuan, L.Y.; Lu, W.F. 3D-printed ceramic triply periodic minimal surface structures for design of functionally graded bone implants. Mater. Design 2020, 191, 108602. [CrossRef]

4. $\quad$ Bai, H.; Cui, Y.; Wang, C.; Wang, Z.; Luo, W.; Liu, Y.; Leng, Y.; Wang, J.; Li, Z.; Liu, H. 3D printed porous biomimetic composition sustained release zoledronate to promote osteointegration of osteoporotic defects. Mater. Design 2020, 189, 108513. [CrossRef]

5. Qiao, S.; Sheng, Q.; Li, Z.; Wu, D.; Zhu, Y.; Lai, H.; Gu, Y.3D-printed Ti6Al4V scaffolds coated with freeze-dried platelet-rich plasma as bioactive interface for enhancing osseointegration in osteoporosis. Mater. Design 2020, 194, 108825. [CrossRef]

6. Krishnakumar, G.S.; Sampath, S.; Muthusamy, S.; John, M.A. Importance of crosslinking strategies in designing smart biomaterials for bone tissue engineering: A systematic review. Mater. Sci. Eng. C Mater. Biol. Appl. 2019, 96, 941-954. [CrossRef]

7. Shie, M.Y.; Shen, Y.F.; Astuti, S.D.; Lee, K.X.; Lin, S.H.; Dwijaksara, N.L.B.; Chen, Y.W. Review of polymeric materials in 4 D printing biomedical applications. Polymers 2019, 11, 1864. [CrossRef]

8. Kang, H.W.; Lee, S.J.; Ko, I.K.; Kengla, C.; Yoo, J.J.; Atala, A. A 3D bioprinting system to produce human-scale tissue constructs with structural integrity. Nat. Biotechnol. 2016, 34, 312-319. [CrossRef]

9. Lai, Y.; Li, Y.; Cao, H.; Long, J.; Wang, X.; Li, L.; Li, C.; Jia, Q.; Teng, B.; Tang, T.; et al. Osteogenic magnesium incorporated into PLGA/TCP porous scaffold by 3D printing for repairing challenging bone defect. Biomaterials 2019, 197, 207-219. [CrossRef]

10. Zhang, W.; Ullah, I.; Shi, L.; Zhang, Y.; Ou, H.; Zhou, J.; Ullah, M.W.; Zhang, X.; Li, W. Fabrication and characterization of porous polycaprolactone scaffold via extrusion-based cryogenic 3D printing for tissue engineering. Mater. Design 2019, 180, 107946. [CrossRef]

11. Lin, Y.H.; Chuang, T.Y.; Chiang, W.H.; Chen, I.W.P.; Wang, K.; Shie, M.Y.; Chen, Y.W. The synergistic effects of graphene-contained 3D-printed calcium silicate/poly- $\varepsilon$-caprolactone scaffolds promote FGFR-induced osteogenic/angiogenic differentiation of mesenchymal stem cells. Mater. Sci. Eng. C Mater. Biol. Appl. 2019, 104, 109887. [CrossRef] [PubMed]

12. Tayebi, L.; Rasoulianboroujeni, M.; Moharamzadeh, K.; Almela, T.K.D.; Cui, Z.; Ye, H. 3D-printed membrane for guided tissue regeneration. Mater. Sci. Eng. C Mater. Biol. Appl. 2018, 84, 148-158. [CrossRef] [PubMed]

13. Chen, C.Y.; Chen, C.C.; Wang, C.Y.; Lee, K.X.; Yeh, C.L.; Lin, C.P. Assessment of the release of vascular

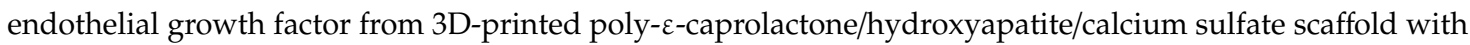
enhanced osteogenic capacity. Polymers 2020, 12, 1455. [CrossRef] [PubMed]

14. Gupta, D.; Singh, A.K.; Kar, N.; Dravid, A.; Bellare, J. Modelling and optimization of NaOH-etched 3-D printed PCL for enhanced cellular attachment and growth with minimal loss of mechanical strength. Mater. Sci. Eng. C Mater. Biol. Appl. 2019, 98, 602-611. [CrossRef]

15. Chiu, Y.C.; Shen, Y.F.; Lee, K.X.; Lin, S.H.; Wu, Y.C.; Chen, Y.W.3D printing of amino resin-based photosensitive materials on multi-parameter optimization design for vascular engineering applications. Polymers 2019, 11, 1394. [CrossRef] 
16. Liu, D.; Nie, W.; Li, D.; Wang, W.; Zheng, L.; Zhang, J.; Zhang, J.; Peng, C.; Mo, X.; He, C. 3D printed PCL/SrHA scaffold for enhanced bone regeneration. Chem. Eng. J. 2019, 362, 269-279. [CrossRef]

17. Chen, Y.W.; Shen, Y.F.; Ho, C.C.; Yu, J.; Wu, Y.H.; Wang, K.; Shih, C.T.; Shie, M.Y. Osteogenic and angiogenic potentials of the cell-laden hydrogel/mussel-inspired calcium silicate complex hierarchical porous scaffold fabricated by 3D bioprinting. Mater. Sci. Eng. C Mater. Biol. Appl. 2018, 91, 679-687. [CrossRef]

18. Shie, M.Y.; Ding, S.J.; Chang, H.C. The role of silicon in osteoblast-like cell proliferation and apoptosis. Acta Biomater. 2011, 7, 2604-2614. [CrossRef]

19. Liu, W.C.; Wang, H.Y.; Chen, L.C.; Huang, S.W.; Wu, C.; Chung, R.J. Hydroxyapatite/tricalcium silicate composites cement derived from novel two-step sol-gel process with good biocompatibility and applications as bone cement and potential coating materials. Ceram. Int. 2019, 45, 5668-5679. [CrossRef]

20. Chen, L.; Deng, C.; Li, J.; Yao, Q.; Chang, J.; Wang, L.; Wu, C. 3D printing of a lithium-calcium-silicate crystal bioscaffold with dual bioactivities for osteochondral interface reconstruction. Biomaterials 2019, 196, 138-150. [CrossRef]

21. Yu, C.T.; Wang, F.M.; Liu, Y.T.; Ng, H.Y.; Jhong, Y.R.; Hung, C.H.; Chen, Y.W. Effect of bone morphogenic protein-2 loaded mesoporous strontium substitution calcium silicate/recycled fish gelatin 3D cell-laden scaffold for bone tissue engineering. Processes 2020, 8, 493. [CrossRef]

22. Wang, J.; Liu, S.; Li, J.; Yi, Z. The role of the fibroblast growth factor family in bone-related diseases. Chem. Biol. Drug Des. 2019, 94, 1740-1749. [CrossRef] [PubMed]

23. Liang, H.; Pun, S.; Wronski, T.J. Bone anabolic effects of basic fibroblast growth factor in ovariectomized rats. Endocrinology 1999, 140, 5780-5788. [CrossRef] [PubMed]

24. Chen, W.; Zhi, M.; Feng, Z.; Gao, P.; Yuan, Y.; Zhang, C.; Wang, Y.; Dong, A. Sustained co-delivery of ibuprofen and basic fibroblast growth factor by thermosensitive nanoparticle hydrogel as early local treatment of peri-implantitis. Int. J. Nanomed. 2019, 14, 1347-1358. [CrossRef] [PubMed]

25. Arunkumar, P.; Dougherty, J.A.; Weist, J.; Kumar, N.; Angelos, M.G.; Powell, H.M.; Khan, M. Sustained release of basic fibroblast growth factor (bFGF) encapsulated polycaprolactone (PCL) microspheres promote angiogenesis in vivo. Nanomaterials 2019, 9, 1037. [CrossRef]

26. Wu, C.; Chang, J.; Fan, W. Bioactive mesoporous calcium-silicate nanoparticles with excellent mineralization ability, osteostimulation, drug-delivery and antibacterial properties for filling apex roots of teeth. J. Mater. Chem. 2012, 22, 16801-16809. [CrossRef]

27. Huang, C.Y.; Huang, T.H.; Kao, C.T.; Wu, Y.H.; Chen, W.C.; Shie, M.Y. Mesoporous calcium silicate nanoparticles with drug delivery and odontogenesis properties. J. Endod. 2017, 43, 69-76. [CrossRef]

28. Huang, K.H.; Chen, Y.W.; Wang, C.Y.; Lin, Y.H.; Wu, Y.H.; Shie, M.Y.; Lin, C.P. Enhanced capability of BMP-2-loaded mesoporous calcium silicate scaffolds to induce odontogenic differentiation of human dental pulp cells. J. Endod. 2018, 44, 1677-1685. [CrossRef]

29. Mandakhbayar, N.; El-Fiqi, A.; Lee, J.H.; Kim, H.W. Evaluation of strontium-doped nanobioactive glass cement for dentin-pulp complex regeneration therapy. ACS Biomater. Sci. Eng. 2019, 5, 6117-6126. [CrossRef]

30. Shen, Y.F.; Ho, C.C.; Shie, M.Y.; Wang, K.; Fang, H.Y. Hinokitiol-loaded mesoporous calcium silicate nanoparticle induce apoptotic cell death through regulation of the function of MDR1 in lung adenocarcinoma cells. Materials 2016, 9, 306. [CrossRef]

31. Domingos, M.; Intranuovo, F.; Russo, T.; De Santis, R.; Gloria, A.; Ambrosio, L.; Ciurana, J.; Bartolo, P. The first systematic analysis of $3 \mathrm{D}$ rapid prototyped poly( $\varepsilon$-caprolactone) scaffolds manufactured through BioCell printing: The effect of pore size and geometry on compressive mechanical behaviour and in vitro hMSC viability. Biofabrication 2013, 5, 045004. [CrossRef] [PubMed]

32. Oh, S.H.; Park, I.K.; Kim, J.M.; Lee, J.H. In vitro and in vivo characteristics of PCL scaffolds with pore size gradient fabricated by a centrifugation method. Biomaterials 2007, 28, 1664-1671. [CrossRef] [PubMed]

33. Bobyn, J.D.; Stackpool, G.J.; Hacking, S.A.; Tanzer, M.; Krygier, J.J. Characteristics of bone ingrowth and interface mechanics of a new porous tantalum biomaterial. J. Bone Joint Surg. Br. 1999, 81, 907-914. [CrossRef] [PubMed]

34. Cheng, C.H.; Chen, Y.W.; Lee, K.X.; Yao, C.H.; Shie, M.Y. Development of mussel-inspired 3D-printed poly (lactic acid) scaffold grafted with bone morphogenetic protein-2 for stimulating osteogenesis. J. Mater. Sci. Mater. Med. 2019, 30, 78. [CrossRef] [PubMed] 
35. Wu, Y.H.; Chiu, Y.C.; Lin, Y.H.; Ho, C.C.; Shie, M.Y.; Chen, Y.W. 3D-printed bioactive calcium silicate/poly- $\varepsilon$-caprolactone bioscaffolds modified with biomimetic extracellular matrices for bone regeneration. Int. J. Mol. Sci. 2019, 20, 942. [CrossRef]

36. Chen, Y.C.; Shie, M.Y.; Wu, Y.H.; Lee, K.X.; Wei, L.J.; Shen, Y.F. Anti-inflammation performance of curcumin-loaded mesoporous calcium silicate cement. J. Formos Med. Assoc. 2017, 116, 679-688. [CrossRef] [PubMed]

37. Huang, K.H.; Lin, Y.H.; Shie, M.Y.; Lin, C.P. Effects of bone morphogenic protein-2 loaded on the 3D-printed MesoCS scaffolds. J. Formos Med. Assoc. 2018, 117, 879-887. [CrossRef]

38. Lin, Y.H.; Chiu, Y.C.; Shen, Y.F.; Wu, Y.H.; Shie, M.Y. Bioactive calcium silicate/poly- $\varepsilon$-caprolactone composite scaffolds 3D printed under mild conditions for bone tissue engineering. J. Mater. Sci. Mater. Med. 2018, 29, 11. [CrossRef]

39. Wang, W.; Liu, D.; Lu, L.; Chen, H.; Gong, T.; Lv, J.; Zhou, S. The improvement of the shape memory function of poly( $\varepsilon$-caprolactone)/nano-crystalline cellulose nanocomposites via recrystallization under a high-pressure environment. J. Mater. Chem. A 2016, 4, 5984-5992. [CrossRef]

40. Chen, T.; Zou, Q.; Du, C.; Wang, C.; Li, Y.; Fu, B. Biodegradable 3D printed HA/CMCS/PDA scaffold for repairing lacunar bone defect. Mater. Sci. Eng. C Mater. Biol. Appl. 2020, 116, 111148. [CrossRef]

41. Liu, X.; Ding, C.; Chu, P.K. Mechanism of apatite formation on wollastonite coatings in simulated body fluids. Biomaterials 2004, 25, 1755-1761. [CrossRef]

42. Ho, C.C.; Fang, H.Y.; Wang, B.; Huang, T.H.; Shie, M.Y. The effects of Biodentine/polycaprolactone 3D-scaffold with odontogenesis properties on human dental pulp cells. Int. Endod. J. 2018, 51, e291-e300. [CrossRef]

43. Liu, C.H.; Hung, C.J.; Huang, T.H.; Lin, C.C.; Kao, C.T.; Shie, M.Y. Odontogenic differentiation of human dental pulp cells by calcium silicate materials stimulating via FGFR/ERK signaling pathway. Mater. Sci. Eng. C Mater. Biol. Appl. 2014, 43, 359-366. [CrossRef] [PubMed]

44. Liu, C.H.; Huang, T.H.; Hung, C.J.; Lai, W.Y.; Kao, C.T.; Shie, M.Y. The synergistic effects of fibroblast growth factor-2 and mineral trioxide aggregate on an osteogenic accelerator in vitro. Int. Endod. J. 2014, 47, 843-853. [CrossRef] [PubMed]

45. Xiao, L.; Esliger, A.; Hurley, M.M. Nuclear fibroblast growth factor 2 (FGF2) isoforms inhibit bone marrow stromal cell mineralization through FGF23/FGFR/MAPK in vitro. J. Bone Miner. Res. 2013, 28, 35-45. [CrossRef] [PubMed]

46. Tcacencu, I.; Rodrigues, N.; Alharbi, N.; Benning, M.; Toumpaniari, S.; Mancuso, E.; Marshall, M.; Bretcanu, O.; Birch, M.; McCaskie, A.; et al. Osseointegration of porous apatite-wollastonite and poly(lactic acid) composite structures created using 3D printing techniques. Mater. Sci. Eng. C Mater. Biol. Appl. 2018, 90, 1-7. [CrossRef] [PubMed]

47. Prins, H.-J.; Braat, A.K.; Gawlitta, D.; Dhert, W.J.A.; Egan, D.A.; Tijssen-Slump, E.; Yuan, H.; Coffer, P.J.; Rozemuller, H.; Martens, A.C. In vitro induction of alkaline phosphatase levels predicts in vivo bone forming capacity of human bone marrow stromal cells. Stem Cell Res. 2014, 12, 428-440. [CrossRef] [PubMed]

48. Xia, Y.; Zhou, P.; Cheng, X.; Xie, Y.; Liang, C.; Li, C.; Xu, S. Selective laser sintering fabrication of nano-hydroxyapatite/poly- $\varepsilon$-caprolactone scaffolds for bone tissue engineering applications. Int. J. Nanomed. 2013, 8, 4197-4213.

49. Fuchs, S.; Ghanaati, S.; Orth, C.; Barbeck, M.; Kolbe, M.; Hofmann, A.; Eblenkamp, M.; Gomes, M.; Reis, R.L.; Kirkpatrick, C.J. Contribution of outgrowth endothelial cells from human peripheral blood on in vivo vascularization of bone tissue engineered constructs based on starch polycaprolactone scaffolds. Biomaterials 2009, 30, 526-534. [CrossRef]

(C) 2020 by the authors. Licensee MDPI, Basel, Switzerland. This article is an open access article distributed under the terms and conditions of the Creative Commons Attribution (CC BY) license (http://creativecommons.org/licenses/by/4.0/). 